POS PROCEEDINGS

\title{
Cosmic dust formation at cryogenic temperatures
}

\author{
Gaël Rouillé ${ }^{* a}$, Serge A. Krasnokutski ${ }^{a}$, Melinda Krebsz ${ }^{b}$, Cornelia Jäger $^{a}$, Friedrich \\ Huisken $^{a}$ and Thomas Henning ${ }^{c}$ \\ ${ }^{a}$ Laboratory Astrophysics Group of the Max Planck Institute for Astronomy at the Friedrich \\ Schiller University Jena, Institute of Solid State Physics \\ Helmholtzweg 3, 07743 Jena, Germany \\ ${ }^{b}$ Institute for Geological and Geochemical Research, Research Centre for Astronomy and Earth \\ Sciences, Hungarian Academy of Sciences \\ 45 Budaörsi street, 1112 Budapest, Hungary \\ ${ }^{c}$ Max Planck Institute for Astronomy \\ Königstuhl 17, 69117 Heidelberg, Germany \\ E-mail: cornelia.jaegereuni-jena.de
}

\begin{abstract}
Within a project aimed at studying the formation of interstellar silicates in dense molecular clouds, we have carried out experiments on the accretion of $\mathrm{SiO}$ molecules at cryogenic temperatures using SiO-doped superfluid He nanodroplets and solid Ne matrices. Mass spectrometry revealed the formation of $\mathrm{Si}_{x} \mathrm{O}_{x}$ oligomers in the doped droplets, i.e., at a temperature of $0.37 \mathrm{~K}$. Therefore the reactions that produce the oligomers have no energy barrier at their entrance channel. Reaction energies were experimentally determined and the results of theoretical calculations were found to be consistent with the measurements. Absorption spectroscopy at UV and mid-IR wavelengths was performed at various stages of the annealing of SiO-doped Ne matrices and after their complete evaporation. It showed that the matrices were actually doped with $\mathrm{Si}_{x} \mathrm{O}_{x}(x=1-3)$ species which disappeared during annealing to be replaced with a condensate characterized by a broad IR spectrum peaking near $9.5 \mu \mathrm{m}$. High-resolution transmission electron microscopy and energy-dispersive X-ray spectroscopy investigations showed that the condensate had on the whole a homogeneous, amorphous structure and the formula SiO. Still, the study of the IR spectra indicated some degree of disproportionation which increased during warming from 8 to $294 \mathrm{~K}$. We have concluded that $\mathrm{SiO}$ molecules can accrete at temperatures as low as $13 \mathrm{~K}$ into a solid compound. These preliminary experiments have demonstrated the relevance of the techniques to the study of accretion at low temperature. More particularly their results allow us to envisage the formation of silicates in the interstellar medium.
\end{abstract}

The Life Cycle of Dust in the Universe: Observations, Theory, and Laboratory Experiments 18-22 November, 2013

Taipei, Taiwan

\footnotetext{
* Speaker.
} 


\section{Introduction}

Silicates are a major component of interstellar dust [1]. As they may grow in dense molecular clouds where temperatures are of the order of $10 \mathrm{~K}$ [2], we have started a project to study the formation and growth of silicates at cryogenic temperatures. In a first stage we have investigated the condensation of $\mathrm{SiO}$ molecules, which represent a major gas-phase carrier of the element $\mathrm{Si}$ in dense clouds [3]. Two techniques have been employed depending on the size of the condensation products. The formation of $\mathrm{Si}_{x} \mathrm{O}_{x}(x=2-3)$ oligomers has been studied using $\mathrm{SiO}$-doped superfluid $\mathrm{He}$ droplets, while that of micrometer-sized $\mathrm{SiO}$ grains has been examined by annealing and evaporating $\mathrm{SiO}$-doped Ne matrices.

Since the results of the experiments with doped He nanodroplets have been reported and analyzed in detail in Ref. [4], they are only briefly presented here. This work focuses on our experiments with doped Ne matrices, more specifically on the characterization of the $\mathrm{SiO}$ grains produced in these experiments. It closes with conclusions on the possible formation of silicates in the ISM.

\section{Experimental}

We have conducted experiments on the condensation of $\mathrm{SiO}$ at cryogenic temperatures using two media, namely, He nanodroplets and Ne matrices. The He nanodroplets are superfluid and have a characteristic temperature of $0.37 \mathrm{~K}$. In contrast, the Ne matrices are solid and can be formed at temperatures as high as $\sim 7 \mathrm{~K}$. They can be annealed to allow the diffusion of dopants, though within a limited range of temperatures and with losses due to evaporation, since pure $\mathrm{Ne}$ sublimates at $\sim 8 \mathrm{~K}[5]$.

In a dedicated vacuum apparatus, He nanodroplets were formed into a beam and directed toward an oven in which $\mathrm{SiO}$ powder was heated up. As they passed through the oven, the nanodroplets picked up $\mathrm{SiO}$ molecules released by the heated powder. In general, molecules are rapidly embedded in the He nanodroplets and cooled down to $0.37 \mathrm{~K}$. Thus, any reaction between pickedup species takes place at this temperature, indicating that its entrance channel does not have an energy barrier. While mass spectrometry is generally applied to detect the revealing products of the reaction, it can also be used to determine the energy of the reaction. A detailed description of the experimental procedures is given in Ref. [4].

When working with Ne matrices, $\mathrm{SiO}$ molecules were produced by shooting at an $\mathrm{SiO}$ pellet with a pulsed laser source. It emitted photons with a wavelength of $266 \mathrm{~nm}$ in pulses of $5 \mathrm{~ns}$ duration at a rate of $10 \mathrm{~Hz}$. The pulses carried an average energy of $\sim 1.7 \mathrm{~mJ}$. As repeated shots formed a hole $0.45 \mathrm{~mm}$ in diameter at the surface of the target, the laser beam was shifted every minute so as to avoid drilling through the target. Together with an excess of Ne atoms, the vaporized species were deposited onto a $\mathrm{KBr}$ substrate placed $\sim 55 \mathrm{~mm}$ away in front of the target and kept at $6.3 \mathrm{~K}$ through the action of a cryocooler. The Ne flow was set to 5 standard cubic centimeters per minute. Thus, Ne matrices were formed that contained isolated $\mathrm{SiO}$ molecules as well as oligomers, as verified by absorption spectroscopy in the UV and mid-IR domains. Reactions between the embedded $\mathrm{SiO}$ molecules and oligomers were made possible by annealing the matrices until their complete evaporation. This was done by heating the substrate gradually up to $13 \mathrm{~K}$. Spectra were measured during the annealing procedure and also while the substrate warmed 

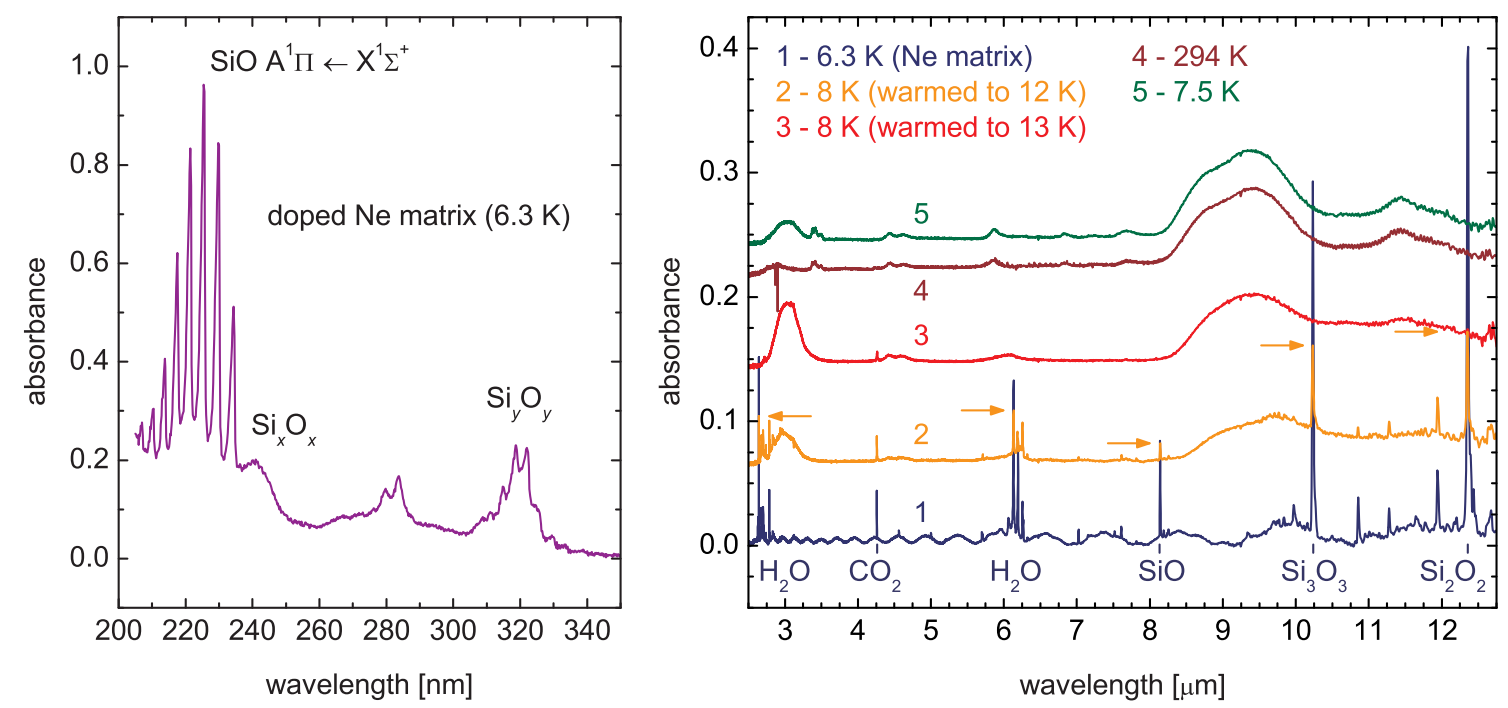

Figure 1: (Left) Ultraviolet absorption spectrum of a Ne matrix doped with $\mathrm{SiO}$ molecules and oligomers. It was measured after depositing material for $11 \mathrm{~min}$. (Right) Infrared absorption spectrum of the same matrix after 31 min of growth, with spectra measured at different temperatures after partial and complete evaporation of the Ne atoms. The sequence of measurements is indicated with numbers. In spectrum number 2 , arrows mark band maxima. A vertical offset is applied to the spectra for clarity.

up to room temperature. Measurements at wavelengths longer than $12.75 \mu \mathrm{m}$ were obscured by noise and could not be used.

\section{Results and discussion}

\subsection{Experiments with He nanodroplets}

The results of the experiments in which He nanodroplets were doped with $\mathrm{SiO}$ molecules have been presented in detail in Ref. [4]. Briefly, the formation of $\mathrm{Si}_{x} \mathrm{O}_{x}$ compounds was observed and attributed to reactions that took place inside the nanodroplets, not only between $\mathrm{SiO}$ molecules, but also between $\mathrm{SiO}$ molecules and oligomers after the latter were formed. Thus, the absence of an energy barrier at the entrance channel of the corresponding reactions has been inferred. Moreover, reaction energies of 178 and $291 \mathrm{~kJ} \mathrm{~mol}^{-1}$ have been experimentally determined for the reactions $\mathrm{SiO}+\mathrm{SiO} \rightarrow \mathrm{Si}_{2} \mathrm{O}_{2}$ and $\mathrm{SiO}+\mathrm{Si}_{2} \mathrm{O}_{2} \rightarrow \mathrm{Si}_{3} \mathrm{O}_{3}$, respectively. The values are consistent with the results of theoretical calculations in which a cyclic structure was given to the oligomers [4].

\subsection{Experiments with Ne matrices}

Concerning our experiments with $\mathrm{Ne}$ matrices, the initial presence of isolated $\mathrm{SiO}$ molecules and oligomers in the solid Ne medium was verified by absorption spectroscopy as illustrated with Fig. 1 (see also Ref. [4]). In the UV domain, isolated $\mathrm{SiO}$ molecules were identified by measuring the $A^{1} \Pi \leftarrow X^{1} \Sigma^{+}$band system [6]. Other features at UV wavelengths were tentatively attributed to $\mathrm{Si}_{x} \mathrm{O}_{x}$ compounds as their intensity increased at the beginning of the annealing procedure, before decreasing [4]. In fact, the presence of $\mathrm{SiO}$ oligomers, formed during deposition, could be 
expected, as reported by Khanna et al. in a similar study with $\mathrm{N}_{2}$ matrices [7]. Accordingly, measurements in the mid-IR region revealed the presence of the $\mathrm{SiO}, \mathrm{Si}_{2} \mathrm{O}_{2}$, and $\mathrm{Si}_{3} \mathrm{O}_{3}$ species (Fig. 1). They were identified using the assignments made by Khanna et al. [7].

Figure 1 shows that, after a first annealing of a doped matrix and the partial evaporation of the $\mathrm{Ne}$ atoms, the sharp lines of the isolated molecules and oligomers had lost their intensity. At the same time, a broad feature had appeared, extending from $\sim 8.2 \mu \mathrm{m}$ toward longer wavelengths. After complete evaporation of the $\mathrm{Ne}$ atoms at $13 \mathrm{~K}$, and cooling to $8 \mathrm{~K}$, the broad feature remained. The maximum of absorbance corresponds to a band peaking at $\sim 9.5 \mu \mathrm{m}$. It is accompanied by a secondary maximum at $\sim 11.4 \mu \mathrm{m}$. We attribute this band to a solid condensate formed by the accretion of the $\mathrm{Si}_{x} \mathrm{O}_{x}$ species.

After warming up to $294 \mathrm{~K}$, a shoulder could be clearly discerned at $\sim 8.7 \mu \mathrm{m}$ on the side of the strongest band and the secondary maximum at $\sim 11.4 \mu \mathrm{m}$ became sharper. Around $10.7 \mu \mathrm{m}$, however, the absorbance decreased. To verify that these changes were not caused by a simple dependence of the spectral properties of the condensate on the temperature, we cooled it back down to $7.5 \mathrm{~K}$, without breaking the vacuum. The spectrum remained essentially the same as it was at $294 \mathrm{~K}$ with the shoulder still visible. Consequently, we have attributed the evolution of the band profile upon warming to a structural modification, e.g., the disproportionation of $\mathrm{SiO}$, in addition to the disappearance of the underlying libration band of water ice. This band extends from $10 \mu \mathrm{m}$ toward longer wavelengths and peaks at $12.8 \mu \mathrm{m}$ (see, for instance, Ref. [8]). It should disappear like the other bands of water ice seen at 3.05 and $6.04 \mu \mathrm{m}$ in the low-temperature spectrum.

The present spectra are in agreement with those obtained by Khanna et al. [7] by annealing a similarly doped $\mathrm{N}_{2}$ matrix from 32 to $50 \mathrm{~K}$. The comparison with literature data reveals that the condensate gives a spectrum similar to that of $\mathrm{Si}_{2} \mathrm{O}_{3}$, which is characterized by a band at $11.4 \mu \mathrm{m}[9,10,11]$. Considering the spectrum measured at room temperature, an attribution of the corresponding peak to silanol groups $(\mathrm{Si}-\mathrm{OH})$ would not be consistent with the weak $\mathrm{OH}$ stretching feature observed between 2.5 and $3.3 \mu \mathrm{m}$ [11]. On the other hand, a contribution from the bending modes of oxidized $\mathrm{SiH}$ groups, the presence of which is indicated by the small bands at $4.5 \mu \mathrm{m}$, is not to be neglected [12].

Following each experiment, we observed the presence of white, micrometer-sized grains on the substrate. The larger grains actually looked like film fragments. As revealed by high-resolution transmission electron microscopy (HRTEM) and energy-dispersive X-ray (EDX) spectroscopy, the condensed material had a homogeneous, amorphous structure with a composition close to SiO. A HRTEM image is displayed in Fig. 2. The two techniques, however, have limitations and our equipment would not allow us to distinguish amorphous concentrations of $\mathrm{Si}$ atoms or even crystalline domains if the latter were as small as $1 \mathrm{~nm}$. The question arises because commercial $\mathrm{SiO}$, such as the powder we pressed to prepare our pellets, has been found to be disproportionated into $\mathrm{Si}$ and $\mathrm{SiO}_{2}$ nanometer-sized domains $[13,14]$.

In the absence of other data, whether the accretion of $\mathrm{SiO}$ molecules and oligomers led to a disproportionated condensate must be found out by examining the IR spectra. One observes that the band at $9.5 \mu \mathrm{m}$ coincides in position with the transverse and longitudinal modes of the $\mathrm{Si}-$ O-Si asymmetric stretching vibrations observed in amorphous $\mathrm{SiO}_{2}$ (see, for instance, Ref. [15]). Moreover, we have noted the $\mathrm{Si}_{2} \mathrm{O}_{3}$ character of the condensate. Consequently, we infer that, just after formation, still at low temperature, the condensate is disproportionated to some extent. The 


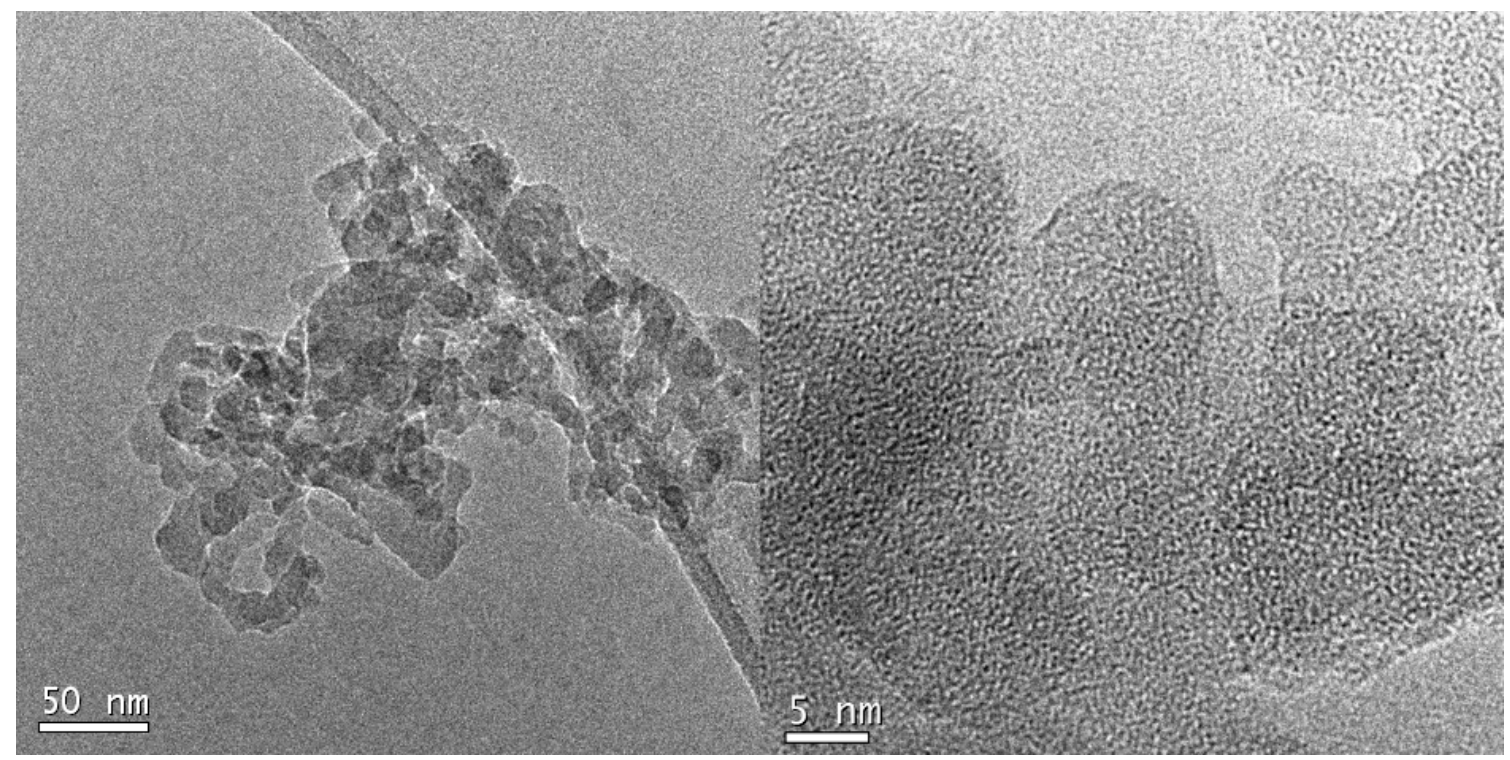

Figure 2: HRTEM images of a grain from an $\mathrm{SiO}$ condensate obtained by annealing and evaporating an SiO-doped Ne matrix. The grain agglomerates have a fluffy morphology and the solid structure appears as homogeneous and amorphous within the limits of the technique.

variations in the spectrum upon warming to room temperature are interpreted as the result of a further disproportionation of the condensate, with a strengthening of the $\mathrm{Si}_{2} \mathrm{O}_{3}$ character.

\section{Conclusions}

In dense molecular clouds, the condensation of silicates would proceed through the accretion of cold gas-phase species onto the even colder surface of small grains acting as seeds. In our experiments, the accreting species are initially hot because of the vaporization procedure. By isolating them in He nanodroplets and in Ne matrices, we cool them to relevant temperatures before they can interact. In addition, we gain the opportunity to verify the nature of the vaporized species. We have found that $\mathrm{Si}_{x} \mathrm{O}_{x}(x=2,3)$ oligomers are obtained through barrierless reactions between $\mathrm{SiO}$ molecules. Moreover, these molecules can accrete into a solid, amorphous compound, forming strong chemical bonds at temperatures as low as $13 \mathrm{~K}$. Thus, interstellar $\mathrm{SiO}$ molecules can be expected to accrete, likely contributing to the formation of silicates since the fingerprint of interstellar $\mathrm{SiO}$ grains, disproportionated or not, has not been reported. Other $\mathrm{O}$ atom-carrying species must accrete along with $\mathrm{SiO}$ to obtain the Si-to-O ratio of silicates. Of course, $\mathrm{Mg}$ and $\mathrm{Fe}$ atoms must also be caught in the accretion process. All the necessary substances are abundant in molecular clouds. The next question to answer is how foreign elements and substances, especially the abundant carbonaceous species, are prevented from interfering with the formation of silicates. It can be proposed that these species that get adsorbed on the surface of a growing silicate are desorbed rapidly enough not to interfer with the growth process. While the substances forming the silicates form relatively strong bonds, the other substances must be weakly attached, allowing their efficient removal, for instance, by the action of interstellar UV photons. Consequently, the cold 
condensation of silicates may already take place in diffuse clouds with low temperature as well as in low-density regions of molecular clouds.

\section{Acknowledgments}

This work was supported by the Deutsche Forschungsgemeinschaft through project No. He 1935/26-1 of the Priority Program 1573 "Physics of the Insterstellar Medium". M. K. is grateful for the award of an Eötvös Scholarship of the Hungarian State.

\section{References}

[1] Henning, T., Cosmic silicates, 2010, ARA\&A, 48, 21

[2] Herbst, E., The chemistry of interstellar space, 2001, Chem. Soc. Rev., 30, 168

[3] Herbst, E., Millar, T. J., Wlodek, S., \& Bohme, D. K., The chemistry of silicon in dense interstellar clouds, 1989, A\&A, 222, 205

[4] Krasnokutski, S. A., Rouillé, G., Jäger, C., Huisken, F., Zhukovska, S., \& Henning, Th., Formation of silicon oxide grains at low temperature, 2014, ApJ, 782, 15

[5] Meier, J., Wittich, G., Classen, J., \& Hunklinger, S., Desorption behavior of quench-condensed argon-neon mixtures, 2002, Phys. Rev. Lett., 88, 016104

[6] Hormes, J., Sauer, M., \& Scullman, R., The vacuum ultraviolet absorption spectrum of SiO in rare gas matrices, 1983, J. Mol. Spectrosc., 98, 1

[7] Khanna, R. K., Stranz, D. D., \& Donn, B., A spectroscopic study of intermediates in the condensation of refractory smokes: Matrix isolation experiments of SiO, 1981, J. Chem. Phys., 74, 2108

[8] Öberg, K. I., Fraser, H. J., Boogert, A. C. A., Bisschop, S. E., Fuchs, G. W., van Dishoeck, E. F., \& Linnartz, H., Effects of $\mathrm{CO}_{2}$ on $\mathrm{H}_{2} \mathrm{O}$ band profiles and band strengths in mixed $\mathrm{H}_{2} \mathrm{O}: \mathrm{CO}_{2}$ ices, 2007, A\&A, 462, 1187

[9] Ritter, E., Zur Kenntnis der $\mathrm{SiO}$ - und $\mathrm{Si}_{2} \mathrm{O}_{3}$-Phase in dünnen Schichten, 1962, Optica Acta, 9, 197

[10] Day, K. L., \& Donn, B., Condensation of nonequilibrium phases of refractory silicates from the vapor, 1978, Science, 202, 307

[11] Hagenmayer, R. M., Friede, B., \& Jansen, M., Structural studies on amorphous $\mathrm{Si}_{2} \mathrm{O}_{3}$ and $\mathrm{H}_{2} \mathrm{Si}_{2} \mathrm{O}_{4}$ by means of diffraction using high energy photons, 1998, J. Non-Cryst. Solids, 226, 225

[12] Tsu, D. V., Lucovsky, G., \& Davidson, B. N., Effects of the nearest neighbors and the alloy matrix on SiH stretching vibrations in the amorphous $\mathrm{SiO}_{r}: \mathrm{H}(\mathrm{O}<r<2)$ alloy system, 1989, Phys. Rev. B, 40, 1795

[13] Hohl, A., Wieder, T., van Aken, P. A., Weirich, T. E., Denninger, G., Vidal, M., Oswald, S., Deneke, C., Mayer, J., \& Fuess, H., An interface clusters mixture model for the structure of amorphous silicon monoxide (SiO), 2003, J. Non-Cryst. Solids, 320, 255

[14] Schulmeister, K., \& Mader, W., TEM investigation on the structure of amorphous silicon monoxide, 2003, J. Non-Cryst. Solids, 320, 143

[15] Gunde, M. K., Vibrational modes in amorphous silicon dioxide, 2000, Physica B, 292, 286 